\title{
Estimation of Shoreline Positions by Combining X-band Radar and SAR Observations
}

\author{
Dipankar KUMAR ${ }^{1}$ and Satoshi TAKEWAKA ${ }^{2}$ \\ ${ }^{1}$ Graduate School of Systems and Information Engineering, University of Tsukuba, \\ (1-1-1 Tennodai, Tsukuba, Ibaraki, 305-8573, Japan) \\ E-mail: dks.bsmrstu@gmail.com \\ ${ }^{2}$ Professor, Dept. of Engineering Mechanics and Energy, University of Tsukuba, \\ (1-1-1 Tennodai, Tsukuba, Ibaraki, 305-8573, Japan) \\ E-mail: takewaka@kz.tsukuba.ac.jp
}

\begin{abstract}
Shoreline positions of Kashima Coast facing the Pacific Ocean, which is approximately $16 \mathrm{~km}$ long with Hasaki Fishery Port at the south end and Kashima Port at the north end, have been observed with four landbased X-band radars and Synthetic Aperture Radar (SAR) satellite. X-band radars observe shoreline positions continuously in time but do not cover the whole coast. On the other hand, SAR covers the whole spatial domain, but data is available only a few times in a year. The purpose of the present work is to propose a data fusion method which combines different shoreline data observed by X-band radars and SAR satellite with the help of Garcia's method, a Penalized Least Square (PLS) regression based on Discrete Cosine Transform (DCT). Garcia's method is initially applied to shoreline positions dataset derived from $\mathrm{X}$-band radars, and its performance has been checked for this dataset with artificial gaps. Then Garcia's method is executed to combine Radar and SAR shoreline positions dataset together. The data fusion result is verified by survey data, and we confirm that our fusion method performs reasonably well to process shoreline data set.
\end{abstract}

Key Words: Kashima Coast, X-band radar, SAR, Shoreline position, Garcia's data smoothing and filling method, Data fusion

\section{INTRODUCTION}

Understanding and monitoring of shorelines are significantly important for proper beach management. In this regard, there are several shorelines monitoring tools such as situ beach profiling, LIDAR surveys, aerial photography, video camera analysis, satellite imagery, Synthetic Aperture Radar (SAR), landbased X-band radar and so on. There have been many attempts to detect shoreline position from the individual observations. However, a large number of high resolution spatial and temporal coverage data are essential for monitoring the inter- and intra-annual intertidal morphological change and seasonal variations of the morphology. Aerial and satellite imaging typically have broad spatial coverage, but their temporal coverage is limited. X-band radar can provide frequent data. However, it has some shortcomings, e.g., limited spatial coverage compared to aerial and satellite imaging, and cost of data processing to digitize shoreline position from the images.

In this context, a data fusion technique is tested here to overcome these types of shortcomings. Data fusion, which integrates multiple datasets from different sources and produces a unified output that preserves the desired information. The benefits of data fusion usually include improved measurement reliability and information completeness (for example, increased spatial coverage or measuring range).

Deronde et al. ${ }^{1}$ used a combination of airborne LIDAR and airborne hyperspectral data to study the beach morphodynamics of the Belgian backshore and foreshore. These authors explain that the combined interpretation of the erosion/sedimentation map with the classified hyperspectral data yields an appropriate method for studying the process of sand transport along the coastline.

The main purpose of this paper is to propose a data fusion method which combines different shoreline data observed by land-based X-band radars and SAR satellite. The data are combined by means of Garcia's method $^{2)}$, a Penalized Least Square (PLS) regression based on Discrete Cosine Transform (DCT). We check the validity of the proposed method by comparing the estimated results with survey data.

\section{DATA}

In this study, two types of data from different methods are used: land-based X-band radar $^{3)}$ and SAR satellite observation ${ }^{4}$. Hourly time-averaged Xband radar images are collected from Dec 1, 2009 to 


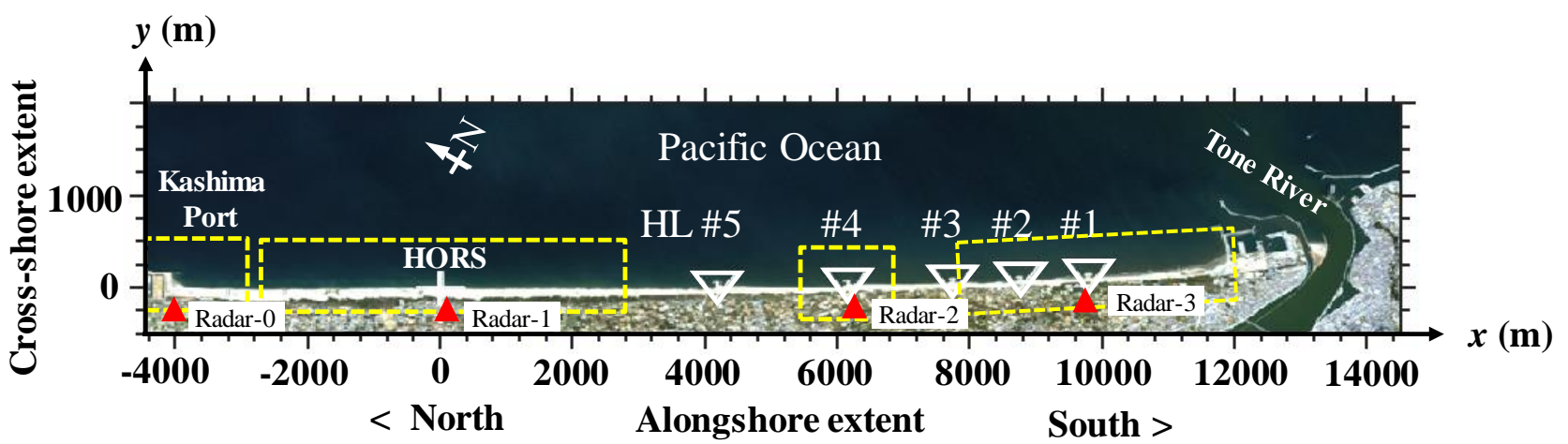

Fig.1 Kashima Coast, Japan. $\Delta$ Radar location. $\nabla$ Headlands (HL, 1-5). The origin of the coordinate system is located at the base of research pier HORS. The rectangular boxes with yellow lines indicate the coverage of radar observations.

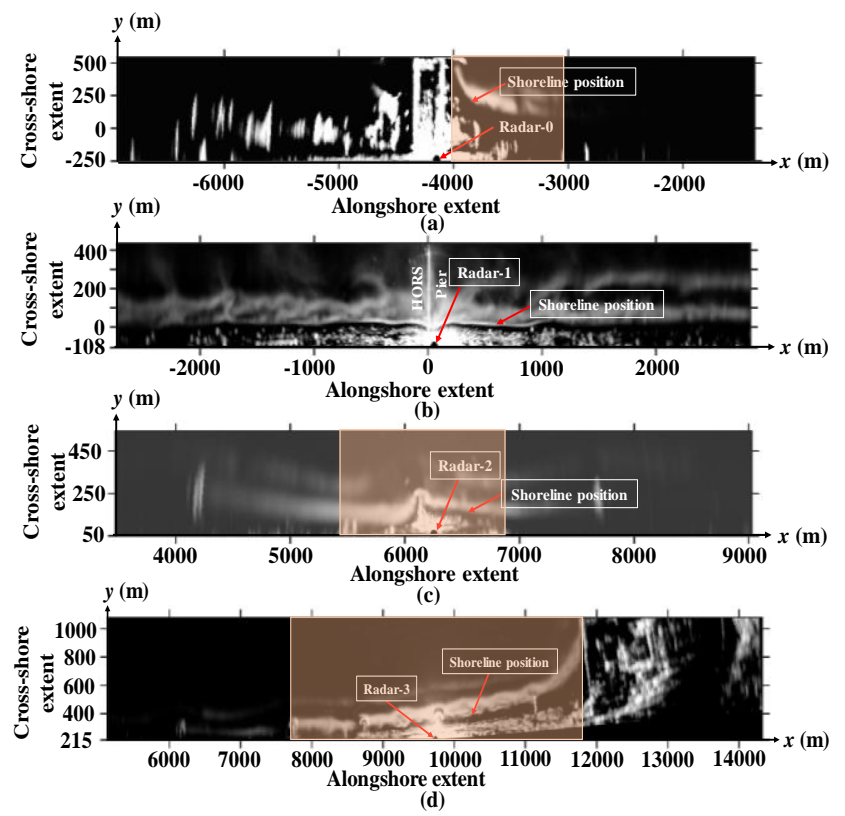

Fig.2 Example of time-averaged X-band radar images captured by (a) Radar-0, (b) Radar-1, (c) Radar-2, and (d) Radar-3, respectively. The shaded area indicates effective spatial coverage of radar observations.

May 15, 2012 with some temporal and spatial gaps, and six SAR satellite data which are captured in 2010 (Jan 30, May 2, Aug 2, Nov 20) and 2011 (Feb 2, April 7).

The study area of the present research is southern Kashima Coast, Japan which is approximately $16 \mathrm{~km}$ long straight sandy coast with Hasaki Fishery Port at the south end and Kashima Port at the end of the north (Fig.1). For the observation of shoreline positions, four land-based X-band radars have been installed, namely Radar-0, Radar-1, Radar-2, and Radar-3. Figure 2 shows the example of hourly time averaged $\mathrm{X}$-band radar images captured by Radar-0, Radar-1, Radar-2, and Radar-3, respectively. The pixel size of Radar-0, Radar-1, and Radar-2 is approximately 5.42 $\mathrm{m}$, and Radar-3 is about $7.18 \mathrm{~m}$. The shaded areas in the panels represent the effective spatial coverage of radar observations. We have three fixed spatial gaps along the coast where no X-band radar data is availa- ble (Fig.1). To cover the whole coast, we have afterwards introduced SAR data which covers the whole domain.

Temporal Waterline Method (TWM) ${ }^{5)}$ is used for digitization of shoreline position from hourly timeaveraged X-band radar images. Bell et al. developed this method which detects intertidal shore profile automatically with the help of pixel intensity from time stack X-band radar images and the binary signal of tidal elevations.

Shoreline positions extracted by TWM from Xband radar images have been displayed in Fig.3. In Fig.3, we observe two types of gaps: (i) fixed spatial gaps $(-43<\mathrm{x}<92 \mathrm{~m} ; 6081<\mathrm{x}<6216 \mathrm{~m} ; 7640<\mathrm{x}$ $<7820 \mathrm{~m} ; 9657<\mathrm{x}<9856 \mathrm{~m} ; 11103<\mathrm{x}<11174 \mathrm{~m})$ which are due to saturation of the radar measurement, and limited coverage of radars, and (ii) random gaps which are due to the lack of strong waterline signals of radar images.

During stormy high wave conditions, the shoreline position is shifted landwards by wave set-up and run-up effect. For this reason, we have introduced an empirical wave run-up formula ${ }^{6}$ to correct the wave run-up effect on TWM extracted shoreline position. Estimation of horizontal landward shift of shoreline position due to wave run-up effect has been shown in Fig.4.

For validation $^{7)}$, we present a comparison among TWM, TWM with runup correction, and surveyed shoreline position (2006-2007) at the research pier HORS in Fig.5 (a) and its corresponding variation of wave height is shown in Fig.5 (b). It is clearly seen that the shoreline position tends to shift the landward direction during high wave conditions. Blue, black, and red solid lines represent the survey, TWM, and TWM with run-up corrected shoreline position, respectively. TWM estimation can follow the trend of survey shoreline position; however, there is some systematic gap which is due to the effect of wave runup of the shift of shoreline position. Shoreline position is estimated landwards compared to the surveyed shoreline position. As mentioned above, an empirical wave run-up formula is utilized to reduce these types 


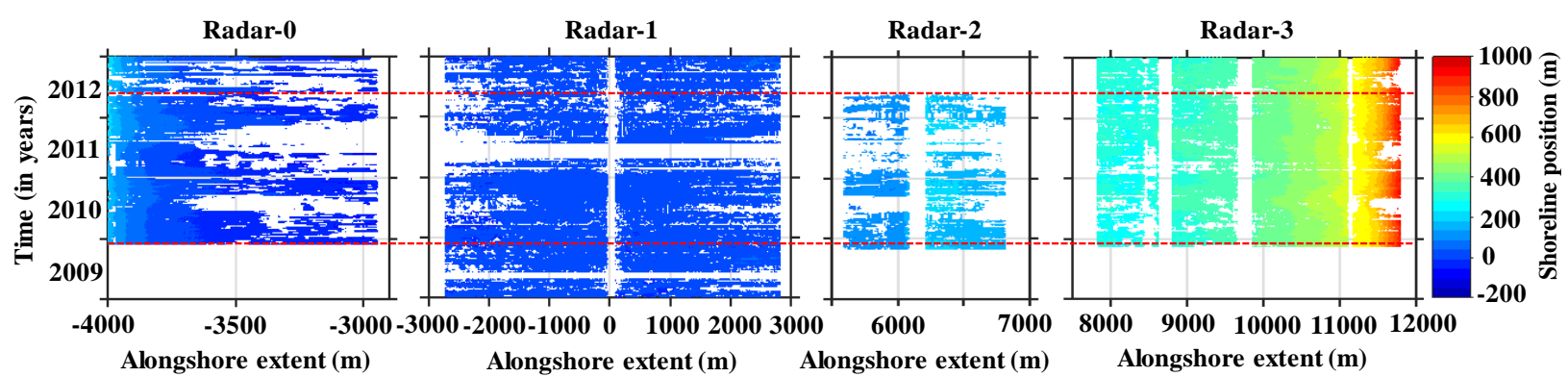

Fig.3 Shoreline positions extracted from radar images by Temporal Waterline Method from the period of 2009 to 2012.

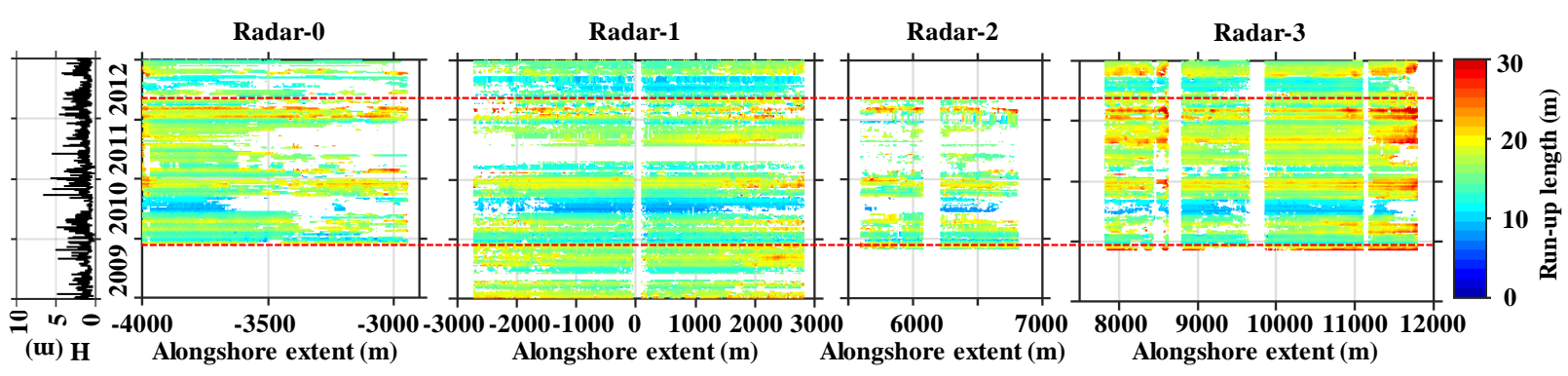

Fig.4 Estimated shift of shoreline positions due to wave run-up effect from the period of 2009 to 2012.
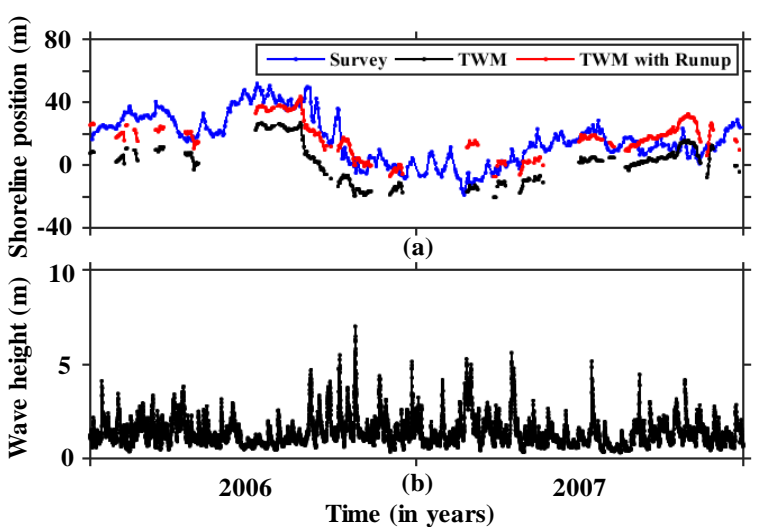

Fig.5 (a)Validation of TWM estimation by comparing with the survey at research pier HORS (2006-2007) (b) variation of significant wave height.

of systematic gap. Consequently, we found the wave run-up corrected TWM shoreline position which can follow the trend of survey shoreline position and minimize the systematic gap on average $8.5 \mathrm{~m}$ by compared to surveyed data.

\section{GARCIA'S DATA FILLING METHOD}

This method is formulated based on a Penalized Least Squares regression method by means of the Discrete Cosine Transform (PLS-DCT), which expresses the data in terms of a sum of cosine functions oscillating at different frequencies, and it is suitable for equally spaced data in one dimension and higher. Since the DCT can be multidimensional, the DCTbased PLS regression can be immediately extended to multidimensional datasets. Wang et al. ${ }^{8)}$ executed the same method and analyzed its performance of filling in data gaps in the global soil moisture dataset.
We will adopt this method to fill random and continuous data gaps for spatio-temporal shoreline datasets.

Now we give a brief introduction of Garcia's algorithm: Let $y$ stand for a spatio-temporal dataset with random or continuous gaps, and $W$ be the diagonal matrix $\operatorname{diag}\left(w_{i}\right)$ that contains the weight $w_{i} \in$ $[0,1]$ corresponding to the data $y_{i}$. In the presence of missing values, $W$ is simply defined by $w_{i}=0$ if $y_{i}$ is missing, while an arbitrary finite value assigned to $y_{i}$. The DCT-PLS seeks for supposed smooth value $\hat{y}$ that minimizes

$$
F(\hat{y})=\left\|W^{1 / 2} \circ(y-\hat{y})\right\|^{2}+s\left\|\nabla^{2} \hat{y}\right\|,
$$

where $\|\|,. \nabla^{2}$, and $\circ$ stand for the Euclidean norm, Laplace operator, and elementwise product, respectively. The $s$ is a positive scalar that controls the degree of smoothing: as $s$ increases, the smoothness of $\hat{y}$ also increases. The $\hat{y}$ can be easily achieved by rewriting Eq. (1) with the type II discrete cosine transform (DCT) and its inverse discrete cosine transform (IDCT), which forms

$$
\hat{y}=\operatorname{IDCT}(\Gamma \circ \operatorname{DCT}(W \circ(y-\hat{y})+\hat{y})) .
$$

Here, the $\Gamma$ is a two-dimensional filtering tensor defined by

$$
\Gamma_{i_{1}, i_{2}}=\left(1+s\left(\sum_{j=1}^{2}\left(2-\cos \frac{\left(i_{j}-1\right) \pi}{n_{j}}\right)\right)^{2}\right)^{-1}
$$

where $i_{j}$ denotes the $i$ th element along the $j$ th dimension, and $n_{j}$ denotes the size of $y$ along this dimension. In Eqs. (2) and (3), the DCT-PLS modeling relies only on the choice of the smoothing parameter $s$. For the purpose of filling in data gaps, this parameter needs to have an infinitesimal value $(\approx 0)$ to reduce the effect of smoothing. A high value of $s$ leads to the loss of high frequency components. 


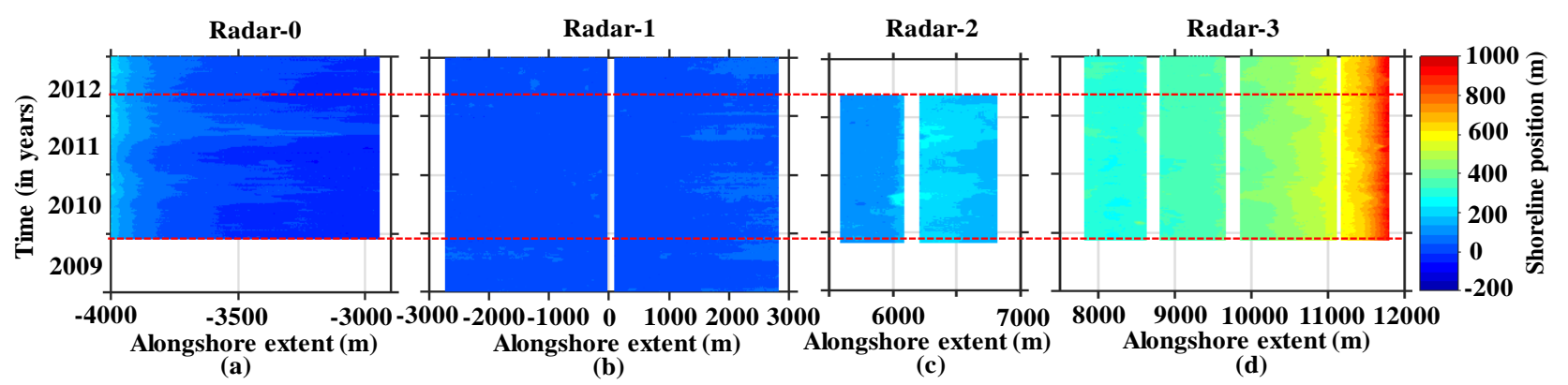

Fig.6 Random gaps in the wave run-up corrected shoreline positions filled by Garcia's method. Red lines represent the common period of four radar observations.
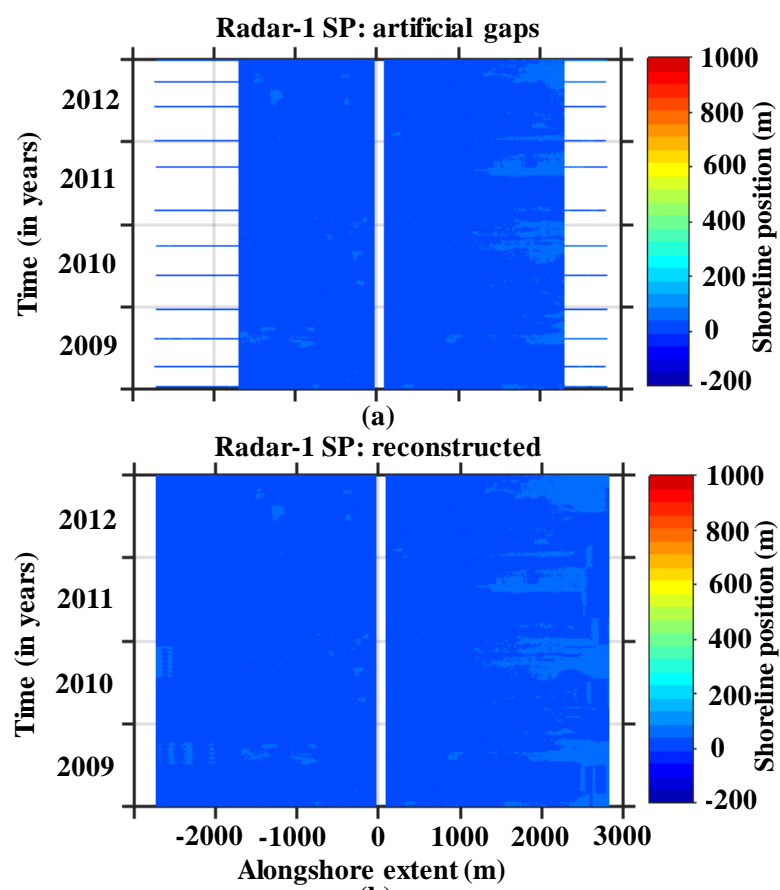

(b)

Fig.7 (a) Artificial temporal gaps set in shoreline positions (Radar-1). Original data is shown in Fig.6(b), and (b) Reconstructed shoreline positions by using Garcia's method.

\section{DATA FILLING AND FUSION RESULTS}

\section{(1) Random gap filling of X-band radar data}

The shoreline position extracted by TWM (Fig.3) combine with wave run-up length (Fig.4) depict the processed radars shoreline position data with numerous random gaps. To fill these random gaps, we applied Garcia's method. The filled radars shoreline position data are shown in Fig.6, which clarifies that the performance of filling is reasonably well from the view of smoothness of shoreline data by taking smoothing parameter value $s=10^{-6}$. That a high value of $s$ leads to the loss of high frequency components. The choice of $s$ is discussed later on.

\section{(2) Validation of Garcia's method}

To validate the performance skill of the Garcia's method, we introduce a $1020 \mathrm{~m}$ long $(-2723<\mathrm{x}<$ $1703 \mathrm{~m})$ and a $527 \mathrm{~m}$ long $(2300<\mathrm{x}<2827 \mathrm{~m})$ artifi-

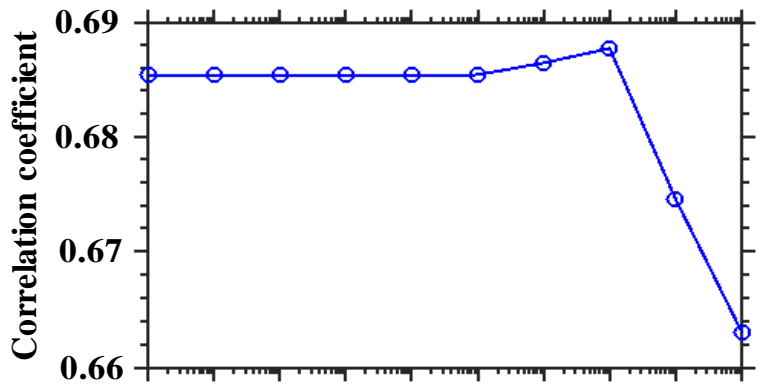

(a)

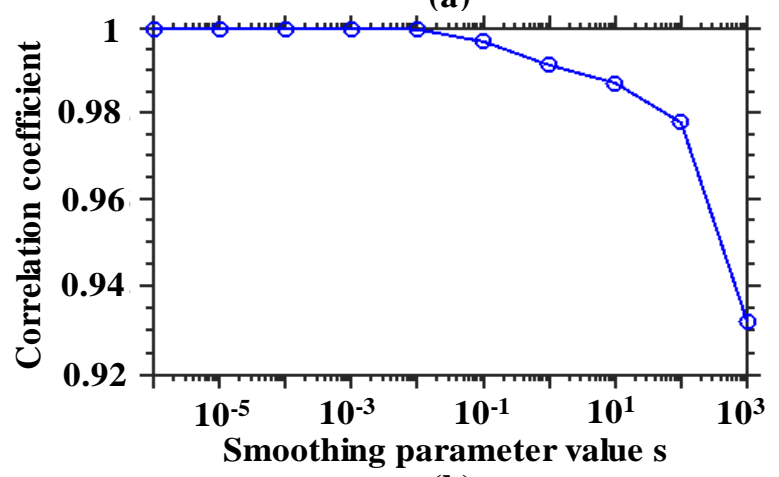

(b)

Fig.8 Significance of smoothing parameter $s$ vs correlation coefficient for gap filling data (a) at $\mathrm{x}=-2360$ $\mathrm{m}$, and for non-gap filling data (b) at $\mathrm{x}=-71 \mathrm{~m}$.

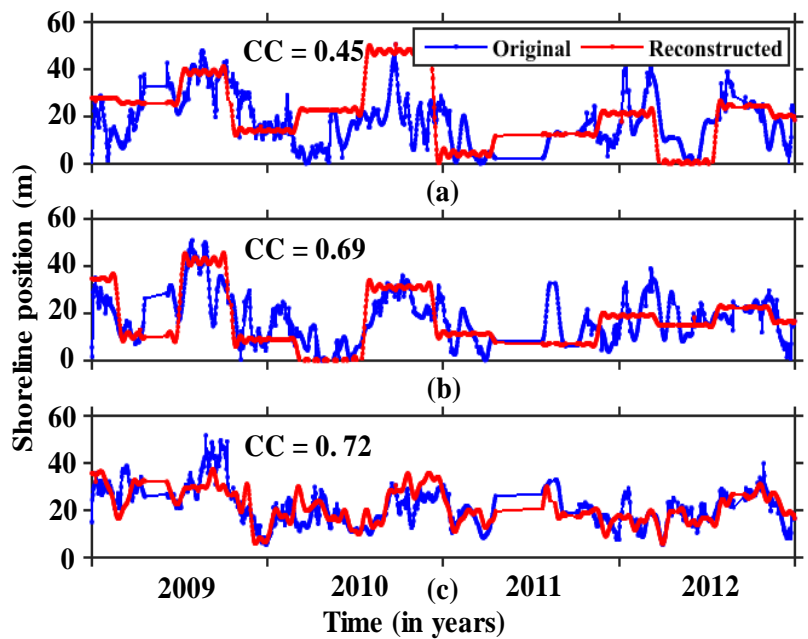

Fig.9 Verification of the performance of data filling by Garcia's method: Comparison of temporal variations of shoreline positions between original and reconstructed data (a) $x=-2702 \mathrm{~m}$, (b) $\mathrm{x}=-2360 \mathrm{~m}$, and (c) $\mathrm{x}=-1801 . \mathrm{m}$ 


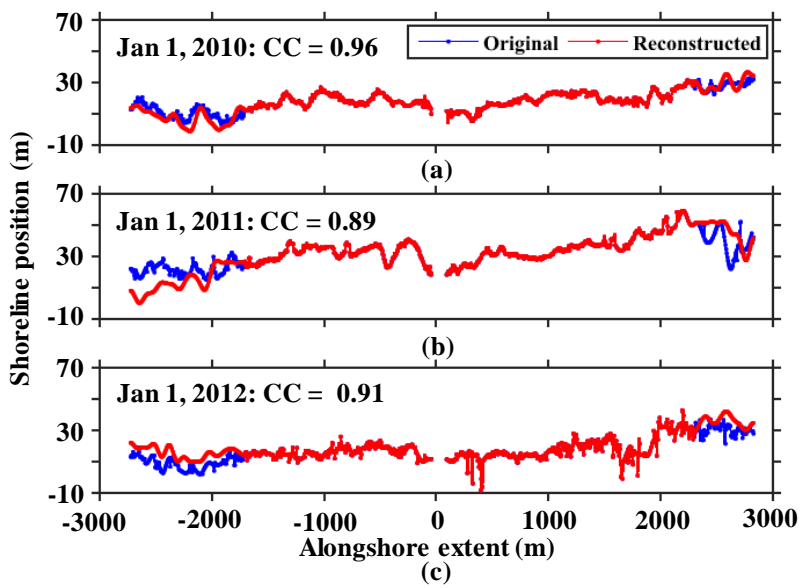

Fig.10 Verification of the performance of data filling by Garcia's method: Spatial distributions of shoreline positions between original and reconstructed data (a) Jan 1, 2010, (b) Jan 1, 2011, and (c) Jan 1, 2012.
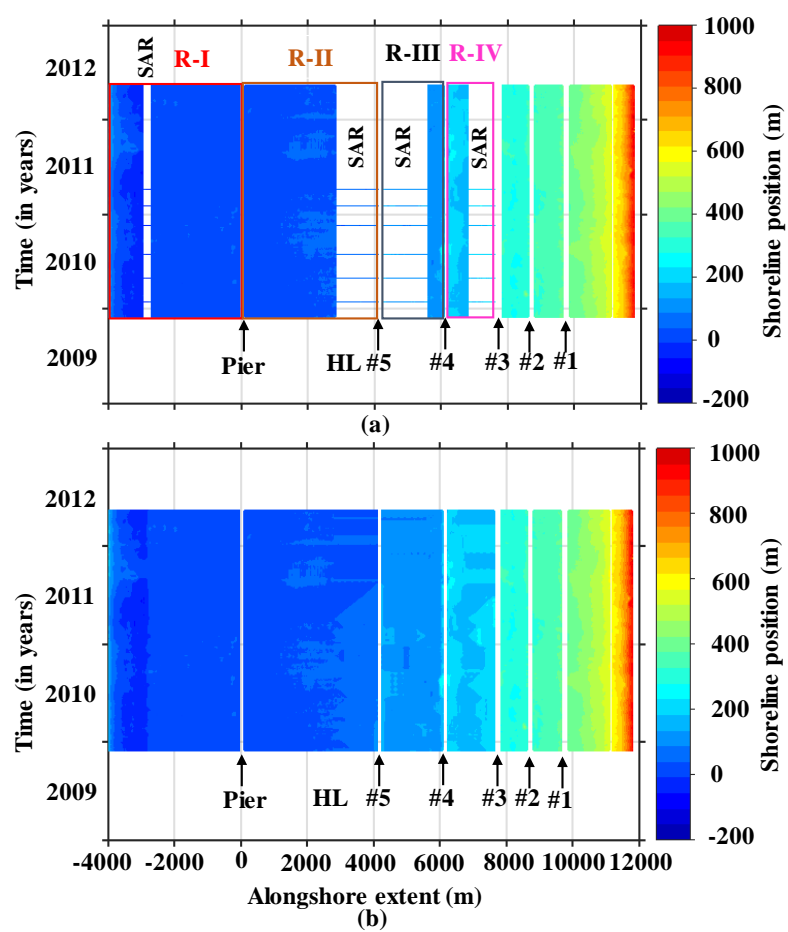

Fig.11 (a) Combination of Radar and SAR shoreline positions data. R-I, R-II, R-III, and R-IV are the subregions of gap filling. (b) The final result of the shoreline variations processed by Garcia's data filling method over the period of Dec 1, 2009 to May 15, 2012.

cial spatio-temporal gaps in the original shoreline dataset of Radar-1 is shown in Fig.7(a). Then Garcia's gap-filling process is applied to this dataset with the choice of smoothing parameter $s=10^{-6}$. The output of gap-filled shoreline data is represented in Fig.7(b).

The choice of smoothing parameter $s$ vs correlation coefficient (CC) for the two different transect at $x=-2360 m$ and $x=-71 m$ is shown in Fig.8. A high correlation coefficient value identifies the strong symmetry between two observations, while low corr-

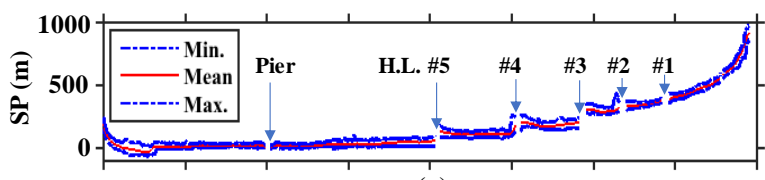

(a)

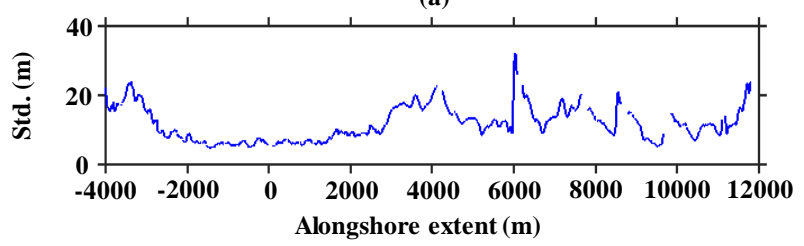

(b)

Fig.12 (a) Mean, minimum and maximum range of the shoreline position variation of fusion data, and (b) their standard deviation of the variation.

elation coefficient is associated with the weak symmetry between the two observations. From Fig.8(a), we see that when smoothing parameter $s$ increases, the correlation coefficients decrease in the case of gap data. For non-gap data, we also found the same tendency which is shown in Fig.8(b).

Figures 9(a), (b) and (c) compare between the temporal variation of the original and reconstructed shoreline positions at $\mathrm{x}=-2702 \mathrm{~m}, \mathrm{x}=-2360 \mathrm{~m}$, and $\mathrm{x}=-1801 \mathrm{~m}$, respectively. In the respective alongshore location, we found the correlation coefficient between the original values and their corresponding predictions are approximately $0.45,0.69$, and 0.72 , respectively. Figures 10(a), (b) and (c) also demonstrate the spatial variation of the original and reconstructed shoreline positions. The correlation factors between original and reconstructed shoreline positions are $0.96,0.89$, and 0.91 on the date of Jan 1 , 2010, Jan 1, 2011, and Jan 1, 2012, respectively. As shown in Fig.10, Garcia's gap-filling method fills in only artificial gap values and unchanged other values.

A good correlation is found when the filled data are close to original data in the case of spatial and temporal variation of shoreline dataset (see Fig.9 and Fig.10). This is suggesting a good prediction skill of Garcia's method that can be expected for filling in data gaps of spatio-temporal shoreline dataset when the gaps are not so large.

\section{(3) Fusion results}

Since the radars do not cover the whole $16 \mathrm{~km}$ coast, we further try to combine six SAR observation shoreline data with radar observation (see Fig.11(a)). Within the SAR spatial coverage, there are fixed gaps $(4120<x<4240 \mathrm{~m})$ due to the existences of Headlands. As shown in Fig.11(a), we set four subregions: $\mathrm{R}-\mathrm{I}(-4000<x<-43 \mathrm{~m})$, $\mathrm{R}-\mathrm{II}(92<x<4120$ $\mathrm{m})$, R-III $(4240<x<6080 \mathrm{~m})$ and R-IV (6216 < $x<$ $7640 \mathrm{~m}$ ) to test the applicability of the present gapfilling technique of the shoreline positions extracted from SAR and radar observations. These four subregions were separated by coastal structures so that 


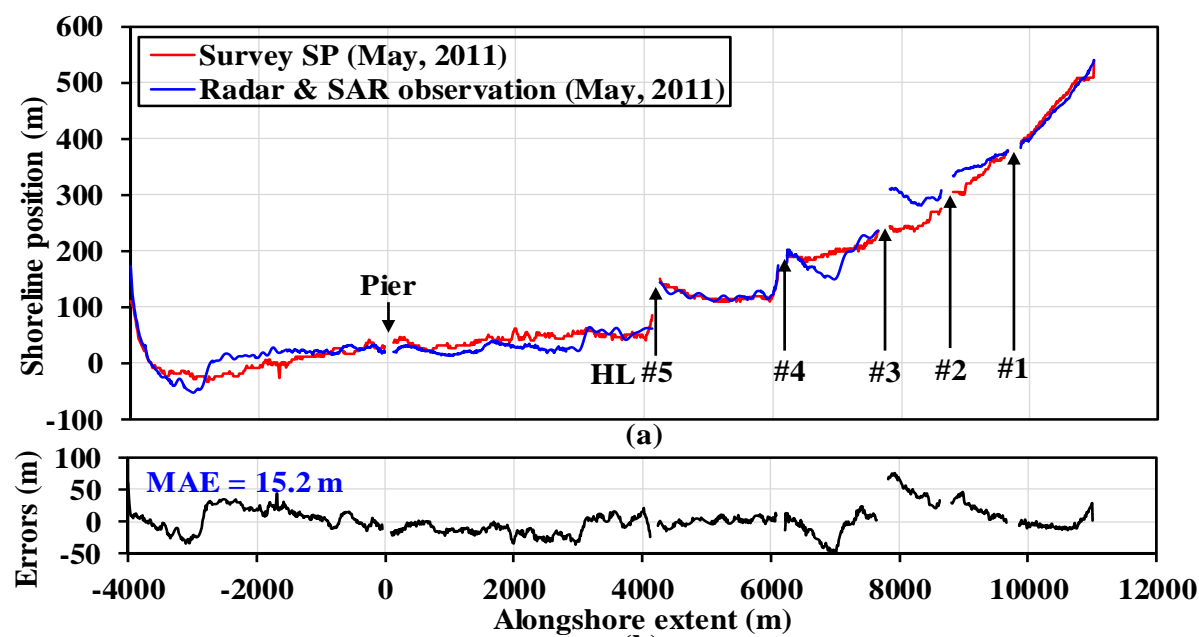

(b)

Fig.13 (a) Validation of the estimated shoreline positions from Radar and SAR observation with survey (2011 May) result, and (b) its corresponding errors.

the shoreline changes in each sub-region shows similar behavior. Again, Garcia's method is applied to fill the numerous data gaps at each sub-region by taking smoothing parameter $s=10^{-6}$, and the result is shown in Fig.11(b).

Figure 12(a) is the mean, maximum and minimum of filled shoreline positions, and Fig.12(b) depicts their standard deviation. Small value of standard deviations and narrow ranges identify stable regions, while large standard deviations and wide envelopes are associated with regions of high variability.

The result of data fusion is verified with survey data. Figure 13(a) compares between shoreline position from the data fusion and survey result and Fig.13(b) represents the difference between the two estimations, mean absolute error (MAE) for the whole region is about $15.2 \mathrm{~m}$. However, we see some large discrepancies greater than $20 \mathrm{~m}$ for $-2810<\mathrm{x}<$ $1400 \mathrm{~m}, 6650<\mathrm{x}<7070 \mathrm{~m}$, and $7800<\mathrm{x}<9200 \mathrm{~m}$. For $2810<x<1400 \mathrm{~m}$, combined shoreline data is basically filled by Garcia's method. Error around the headlands \#2 and \#3 seems significantly large. One possible reason is the low quality of radar images; however, we could not verify it quantitively.

Based on the MAE estimation, we may conclude that our fusion method performs reasonably well to process overall shoreline dataset; however, in some regions the error becomes large.

\section{CONCLUSIONS}

In this paper, we presented a data fusion method to combines the X-band radar and SAR shoreline data with the help of Garcia's data filling and smoothing method. The method is successfully executed, and we verified the result with survey data. Eventually, we may conclude that our proposed data fusion method succeeds reasonably well to process overall shoreline dataset.
ACKNOWLEDGMENT: The radar observations are supported by the members of Littoral Drift Division, and Port and Airport Research Institute. This study is financially supported by the Grants-in-Aid of the Japan Society for the Promotion of Science (JSPS), the Social Implementation Program on Climate Change Adaptation Technology (SI-CAT), and Ministry of Education, Culture, Sports, Science and Technology (MEXT).

\section{REFERENCES}

1) Deronde, B., Houthuys, R., Debruyn, W., Fransaer, D., Lancker, V.V. and Henriet, J.P.: Use of airborne hyperspectral data and laser scan data to study beach morphodynamics along the Belgian coast. Journal of Coastal Research, Vol. 22, pp.1108-1117, 2006.

2) Garcia, D.: Robust smoothing of gridded data in one and higher dimensions with missing values. Comput. Statistical Data Analysis, Vol. 54, pp. 1167-1178, 2010.

3) Takewaka, S.: Measurements of shoreline positions and intertidal foreshore slopes with X-band marine radar. Coastal Engineering Journal, Vol. 47, No. 2 \& 3, pp. 91-107, 2005.

4) Takewaka, S., Matsumoto, A. and Ebihara, Y.: Study on shoreline variabilities of southern Kashimanada coast analyzed from frequently observed SAR data. Journal of $\mathrm{Ja}$ pan Society of Civil Engineers, Series B2, Vol. 74, 2018, (in Japanese with English abstract).

5) Bell, P.S., Bird, C.O. and Plater, A.J. : A temporal waterline approach to mapping intertidal areas using X-band marine radar. Coastal Engineering, Vol. 107, pp. 84-101, 2016.

6) Hasan, G. M. J. and Takewaka, S: Observation of a stormy wave field with X-band radar and its linear aspects, Coastal Engineering Journal, Vol. 49, No. 2, pp. 149-171, 2007.

7) Kumar, D. and Takewaka, S.: Automatic shoreline position and intertidal foreshore slope detection from X-band radar images using Temporal Waterline Method with corrected wave run-up (submitted).

8) Wang, G., Garcia, D., Liu, Y., De Jeu, R. and Dolman, A.J.: A three-dimensional gap filling method for large geophysical datasets: Application to global satellite soil moisture observations. Environmental Modelling \& Software, Vol. 30, pp.139-142, 2012.

(Received March 15, 2018) 